Review Article

\title{
Application of Calculus of Variation in the Optimization of Functional Parameters of Compacted Modified Soils: A Simplified Computational Review
}

\author{
Kennedy C. Onyelowe $\mathbb{D}^{1,2}$ \\ ${ }^{1}$ Department of Mechanical and Civil Engineering, School of Engineering and Applied Sciences, \\ Kampala International University, Kampala, Uganda \\ ${ }^{2}$ Department of Civil Engineering, Michael Okpara University of Agric, Umudike, Nigeria \\ Correspondence should be addressed to Kennedy C. Onyelowe; kennedychibuzor@kiu.ac.ug
}

Received 8 December 2020; Revised 3 March 2021; Accepted 20 April 2021; Published 28 April 2021

Academic Editor: Muhammad Uzair Awan

Copyright (c) 2021 Kennedy C. Onyelowe. This is an open access article distributed under the Creative Commons Attribution License, which permits unrestricted use, distribution, and reproduction in any medium, provided the original work is properly cited.

\begin{abstract}
Fixed endpoint problems (FEPPs) in constrained systems like the effect of curing time or the effect of certain additives in soil stabilization operations have been reviewed illustratively for sustainability purposes in geotechnics. The calculus of variation $(\mathrm{CoV})$ technique of Hamilton's problem was demonstrated using a typical case in geotechnics; the effect of curing time on the unconfined compressive strength of expansive soils is utilized as foundation materials. The era of smart technologies is evolving, and to key into this fast-moving area to help the field of geotechnics, it is required that these new areas are deployed to study their usefulness. The use of $\mathrm{CoV}$ in modeling or simulating geotechnical properties of soil behavior is not prominent and has been played down due to the uncertainties surrounding it. However, this work has identified that if any geotechnical system can be demonstrated in graphs, then the use of CoV becomes easy with the mathematical concept that curves are elements of straight paths. The results of this work show that $\mathrm{CoV}$ is a powerful tool to achieving sustainable optimization of quality properties of stabilized for sustainable and optimal materials handling, design, and construction.
\end{abstract}

\section{Introduction}

The application of numerical and empirical techniques is an evolving method in geotechnical and geoenvironmental engineering. This has also evolved to the deployment of artificial intelligence algorithms in solving many of the everyday problems in earthworks and geotechnics in general as posited by Onyelowe et al. [1,2]. These novel techniques have encouraged smart geotechnics design and the effectiveness of computational geotechnics. Calculus of variation $(\mathrm{CoV})$ is one such smart and numerical method that has helped researchers and engineers to resolve complex engineering problems. Although this method is not commonly and presently evolving in soil mechanics and stabilization, it is the mission of this work to present the possibilities of utilizing this evolving mathematical method, which relies on the graphical behavior of a system to optimize its characteristic components. Fundamental approaches have been identified in the solutions of $\mathrm{CoV}$, and they are known as Hamilton's and Brachistochrone problems $[3,4]$. While Hamilton's method is commonly used in solving most engineering problems, the Brachistochrone method handles systems with the effects of velocity, gravity, friction, and so on. In this overview, Hamilton's method will be used while the other methods will be extensively dealt with in future works. According to Hamilton's method, solving problems in calculus of variations $(\mathrm{CoV})$ is such that the local minima and maxima of the function $f(x)$ of a complex system are identified:

$$
f^{1}(x)=0 .
$$

Further taking the second derivative of the above system of functions, $f(x)$ gives 


$$
f^{11}(x)=0
$$

and the functional of the functions of the above system is given by

$$
I[f(x)]
$$

$\mathrm{CoV}$ according to Hamilton's method moves from functions to real systems while traditional calculus moves from real to real as presented in the following equation:

$$
R-f-R \| f(x)-f-R .
$$

While traditional calculus tries to find the stationary point, $\mathrm{CoV}$ tries to solve the problems of a stationary function, which the distance functional between two points of a system within which the system is optimized. In construction materials and geoenvironmental engineering, earthwork systems behave in a parabolic pattern when exposed to forces or load. For example, the effect of curing time on unconfined compressive strength (UCS), California bearing ratio (CBR), suction (capillary effect), swelling potential, resistance value, and durability as time-dependent system is presented in parabolic graphs. Similarly, during the improvement of these properties through the use of admixtures and supplementary cementitious materials (SCMs), research results have shown that the behavior of these properties in soil/additive blend is always presented in curves. As long as the graphical presentation of an engineering problem like the ones mentioned is parabolic with boundary conditions, the use of $\mathrm{CoV}$ becomes easy. CoV has found applications in different areas of engineering, mathematics, and statistics, and the results have been useful in the optimal control of different systems [3-7]. Modern applications of $\mathrm{CoV}$ have been limited to the areas of biological, biomedical, and materials sciences with remarkable outcomes [8-10]. Differential calculus and integral calculus play a pivotal role in the analysis of variational problems with minima and maxima, and this is emerging with great impact in the field of engineering $[11,12]$. The closest this technique under consideration was applied in geotechnics was on the bearing capacity problem of a foundation on a slopy surface, and this parametric and nondimensional algorithm was greatly useful in resolving the applied force uncertainties of footings on slopes $[13,14]$. Other smart methods have been applied in different protocols of soil stabilization and civil earthworks in general, like Scheffe optimization, extreme vertices, analysis of variance, multiple regression, back-propagation artificial neural network, fuzzy logic, and ANN, and the results have also shown that these numerical, analytical, and statistical methods of soil properties simulation proffer sustainable means of studying the performance of engineering systems [1, 15-17].

Meanwhile, the present work presents a simplified innovative computational review, a type never seen before in the field of computational geotechnics, that promises to open a new innovative and novel approach towards solving and optimizing solutions to geoenvironmental engineering problems in a smarter method. It can be seen and confirmed that the calculus of variation has hardly made it in systematically solving most geotechnical problems fundamentally due to the lack of full understanding of the flexibility of this approach. It will be a new dawn for the field as there will be more robust grounds which are to be opened at the end of this simplified review.

\section{Variational Formulation and Boundary Conditions}

Solving problems in calculus of variation $(\mathrm{CoV})$ is such that a function $f(x)$ is identified in a curve A-B system typical of such presented in Figure 1 with a peak and a deep oriented within the $Y$ - and $X$-axes of a graphical configuration like we encounter in most engineering system behavior.

In order to determine the value of $x$ corresponding to local minimum $\left(x_{\min }\right)$ or in orderwords, the deep in the function graph, conduct the first differential of the function $f(x)$, thus $f^{1}(x)$ or $(\mathrm{d} y / \mathrm{d} x)$, and set it to 0 . It can be noted that the values of $x$ which satisfy this condition are possible values of $x_{\min }$. Because the values of the solution above may not correspond to the local minimum $\left(x_{\min }\right)$, further testing is carried out by using the second derivative of function $f^{1}(x)=0$. The first derivative may not be sufficient to establish the local minimum because it gives stationary points in which this function is not changing. To solve the problem of the function in Figure 1, a step further is required to determine the stationary functions of a functional $I[F]$ (function of functional). In this case, a special equation, usually a differential equation for a stationary function $f(x)$ is formulated and solved. Thus, this second problem is solved using the techniques of $\mathrm{CoV}$, thereby moving from regular calculus to variational calculus (VC) or CoV. When this is achieved in solving engineering problems, the systems of stationary points have moved from regular or traditional calculus to stationary functions in CoV, the function becomes functional equations to solve for stationary points which becomes equation to solve for stationary functions, and an additional layer of deviation is added. To solve for the stationary function within the admissible variation space (AVS), it is shown in Figure 1 to solve for the path linking $A$ and $B$ such that the distance A-B is optimized to its minima values. This seems like a trivial problem because it is a known fact that the shortest path between two points is a straight-line joining the points. Therefore, in this case, the shortest distance with which the system is optimized is the straight line between $\mathrm{A}-\mathrm{B}$ by a common observation. It is required to formulate and solve this by a system of variational calculus, and we learn that the length of a smooth curve $y=f(x)$ between A and B is designated as " $I$ " and is given as follows:

$$
I=\int_{A}^{B} \Delta S
$$

where $\Delta S$ is an element of arch length of the curve.

From Figure 1, a governing equation that shows the changes along the path of a stationary function states that the function $f(x)$ is as follows: 


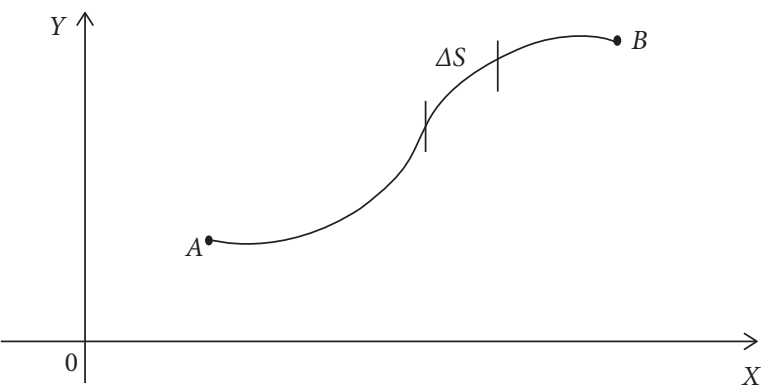

Figure 1: The governing function $f(x)$ of variational problems.

$$
f(x)=\frac{\mathrm{d} y}{\mathrm{~d} x}+\Delta S * \varepsilon_{x},
$$

where $\Delta S$ is the degree of variation and $\varepsilon_{x}$ is the change in function.

By adopting the Pythagoras principle, the degree of variation along the stationary path of a variational problem $\Delta S$ is determined as follows:

$$
\int_{A}^{B} \Delta S^{2}=\sqrt{\mathrm{d} x^{2}+\mathrm{d} y^{2}}
$$

Substituting for $\Delta S$ in equation (5), we get

$$
I=\int_{x_{1}}^{x_{2}} \sqrt{1+\frac{\mathrm{d} y^{2}}{\mathrm{~d} x^{2}}} * \mathrm{~d} x
$$

where $A=x_{1}$ and $B=x_{2}$

To find the path of minimum distance, a CoV problem is formulated [R] as to find $y=f(x)$ between A and B of stationary functions such that the integral $I$ of equation (8) is minimized with respect to $y=f(x)$. The process of minimizing the path of the stationary function equally minimizes other functions on the path and maximizes some others.

To formulate the $\mathrm{CoV}$ from equation (8) requires that a general idea that the following equation is stationary:

$$
I[f]=\int_{x_{1}}^{x_{2}} F\left(x, y, \frac{\mathrm{d} y}{\mathrm{~d} x}\right) * \mathrm{~d} x,
$$

where $F$ is known as the Lagrange, which is an expression involving $x, y$, and $(\mathrm{d} y / \mathrm{d} x)$.

In solving the system that consists of equations (8) and (9), the Euler-Lagrange equation is formulated although the E-L equation does not evaluate the nature of the functionals, and this implies that it does not show whether a functional is minimized or maximized rather it just shows that the functional is stationary.

Taking the partial derivative of equation (6) gives the following equation:

$$
\frac{\partial y_{(x)}}{\partial \Delta S}=0+\frac{\partial \Delta S}{\partial \Delta S} \varepsilon_{x}+\frac{\Delta S \partial}{\partial \Delta S} \varepsilon_{x}
$$

The function in equation (9) is minimized when the degree of variation $(\Delta S)$ is 0 ; thus,

$$
\frac{\mathrm{d} I}{d \Delta S} \mid(\Delta S=0)=0=\int_{x_{1}}^{x_{2}}\left[\frac{\partial F}{\partial y}\left(\frac{\partial y}{\partial \Delta S}\right)+\frac{\partial F}{\partial y^{1}}\left(\frac{\partial y^{1}}{\partial \Delta S}\right)\right] * \mathrm{~d} x,
$$

where

$$
\begin{aligned}
& \frac{\partial y}{\partial \Delta S}=\varepsilon_{x}, \\
& \frac{\partial y^{1}}{\partial \Delta S}=\varepsilon_{x}^{1} .
\end{aligned}
$$

Equation (11) is at the minimum point where the straight path of the system forms a stationary point and zero slope or zero degree of variation $(\Delta S)$. However, equation (11) becomes equation (13) after substituting for equation (12) differential derivative parameters:

$$
\frac{\mathrm{d} I}{d \Delta S} \mid(\Delta S=0)=0=\int_{x_{1}}^{x_{2}}\left[\frac{\partial F}{\partial y} * \varepsilon_{x}+\frac{\partial F}{\partial y^{1}} * \varepsilon_{x}^{1}\right] * \mathrm{~d} x .
$$

From the laws of integrals, equation (13) is estimated by integration by parts solution method by adopting the end conditions; $x_{1=} x_{2=} 0$, and it becomes

$$
\left[\frac{\partial F}{\partial y}-\frac{d}{\mathrm{~d} x}\left(\frac{\partial F}{\partial y^{1}}\right)\right] \varepsilon_{x} * \mathrm{~d} x=0
$$

The minimized path to achieve the optimization (minima and maxima) of the system follows equation (14), where $F$ is the Lagrange or the variation of the system. The Euler-Lagrange equation is the first part of equation (14); thus,

$$
\frac{\partial F}{\partial y}-\frac{d}{\mathrm{~d} x}\left(\frac{\partial F}{\partial y^{1}}\right)=0
$$

This follows that for every engineering problem with system and material characteristics of the form illustrated in Figure 1, such system can be solved by optimizing its functional parts by the method of CoV. With this and from the foregoing, the Lagrange $F$ is given by equation (8) as

$$
F=\sqrt{1+\left(y_{x}^{1}\right)^{2}}
$$

Following boundary conditions of equation (11), 


$$
\begin{aligned}
\frac{\partial F}{\partial y} & =0, \\
\frac{\partial F}{\partial y^{1}} & =\frac{1}{2} \frac{1}{\sqrt{1+\left(y^{1}\right)^{2}}} \\
* 2 y^{1} & =\frac{y^{1}}{\sqrt{1+\left(y^{1}\right)^{2}}} \\
\frac{d}{\mathrm{~d} x}\left(\frac{y^{1}}{\sqrt{1+\left(y^{1}\right)^{2}}}\right) & =0(\text { constant }), \\
\frac{y^{1}}{\sqrt{1+\left(y^{1}\right)^{2}}} & =K .
\end{aligned}
$$

Therefore, substituting the Lagrange $(F)$ in the E-L equation to establish that the minimum path between $\mathrm{A}\left(x_{1}\right)$ and $\mathrm{B}\left(x_{2}\right)$ is a straight path and solving equation (18), gives the following:

$$
\frac{\mathrm{d} y}{\mathrm{~d} x}=a x+b
$$

where equation (19) is a straight-line equation with slope " $a$ " and intercept " $b$ " of the optimized system. This proves that the stationary path, which is the path for which all variational systems are optimized, is a straight line.

\section{Euler-Lagrange Solution of Geotechnics Systems}

During the stabilization of soft soils for earthworks, the quality properties of expansive or problematic soils are treated with binary or ternary or even more components with pozzolanic characteristics to improve the ultimate limit service of soils subjected to cyclic vehicular loads (CVLs). The life service of flexible pavements and of course the pavement foundation relies on the successful stabilization practice. However, then the sustainability of this protocol must be established. Apart from the availability of stabilization components, which are geomaterials at little or no cost for sustainable construction, the management of the material components during and after design, construction, and use is of utmost important. The application of computational geotechnics and of course artificial intelligence (AI) ensures that construction materials are managed for optimum application. Unconfined compressive strength (UCS) of compacted or treated and compacted soils is a major engineering parameter that is pivotal to the assessment of the suitability of soils utilized as compacted subgrade materials. In this illustrative review, the intention is to conduct the calculus of variation formulation of UCS to show the novel approach of using $\mathrm{CoV}$ to solve soil stabilization problems and to optimize the strength of treated soils and the utilization of construction materials as binders in this procedure. In this case, UCS is used interchangeably with $\mathrm{C}$ to designate the unconfined compressive strength. Figure 2 presents a typical case, which shows the effect of curing time $(t)$ in days on the unconfined compressive strength (C) in $\mathrm{kN} / \mathrm{m}^{2}$. For clarity purposes, this pair of quality properties of the soil study could have been obtained with $\mathrm{CBR}$, resilient modulus (MR), resistance value (r-value), compaction, durability, and son on of untreated or treated compacted soils for foundation design and construction. In this, $C$ is maximized against a value of minimized $t$ where A-B is admissible variational space (AVS) of the system with end conditions of $C_{1} t_{1}$ and $C_{2} t_{2}$. Apart from the natural curve $\mathrm{ADB}$ of the system, the system could have also taken another curve path ACB to arrive at $\mathrm{B}$, which is the maximum point of the maximized C. However, within the AVS exists the stationary function which optimizes the system and gives the shortest path between $\mathrm{A}$ and $\mathrm{B}$ at minimum $t$. Figure 3 shows the degree of variation $\delta$ that exists between the natural curve path of the system A-C-B and A-D-B or A-E-B while the optimized path of AVS A-B is the path of stationary function upon which $\mathrm{C}$ is maximized at a minimized $t$.

To solve this optimization problem using Hamilton's solution, the E-L equation of the system illustrated in Figures 2 and 3 has to be formulated and solved to prove that the variational solution of the system depends on the straight path between A and B. Once solved to that extent, the slope and intercept of the straight-line equation become natural boundary conditions for solving the entire CoV system. The variational solution of Figures 2 and 3 is as follows:

$$
C_{(t)}=C_{(t)}^{1}+\delta \varepsilon_{t},
$$

where $C_{(t)}$ is the function of the curve of typical compression-time system, $C_{(t)}^{1}$ is the first partial derivative $(\partial C / \partial t)$ of the compression function, $\delta$ is the degree of variation from the natural curve of the system, and $\varepsilon_{t}$ is the change in function due to variational shifts.

$$
\delta_{(t)}=\int_{t_{1=0}}^{t_{2=28}} \sqrt{1+\left(C_{t}^{1}\right)^{2}} * \mathrm{~d} t
$$

The distance function of the path $\mathrm{A}-\mathrm{B}$ is given as

$$
I[f]=I_{(\delta)}=\int_{t_{1}}^{t_{2}} F\left(t, C_{(t)}, \frac{\partial C}{\partial t}\right) * \mathrm{~d} t .
$$

Formulating the chain equation of the system from equation (21) and equation (22) gives

$$
\frac{\mathrm{d} I}{\mathrm{~d} \delta} \mid(\delta=0)=0=\int_{t_{1}(0)}^{t_{2}(28)}\left[\frac{\partial F}{\partial C}\left(\frac{\partial C}{\partial \delta}\right)+\frac{\partial F}{\partial C^{1}}\left(\frac{\partial C^{1}}{\partial \delta}\right)\right] * \mathrm{~d} t,
$$

where

$$
\begin{gathered}
\frac{\partial C}{\partial \delta}=\varepsilon_{t}, \\
\frac{\partial C^{1}}{\partial \delta}=\varepsilon_{t}^{1} .
\end{gathered}
$$




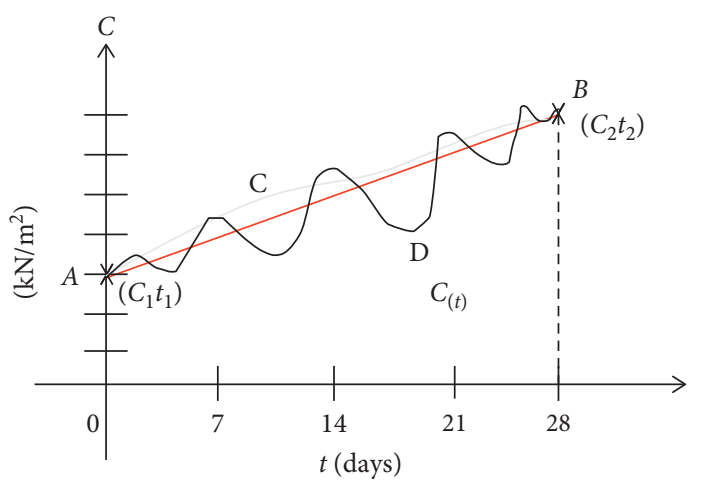

Figure 2: Admissible variational space of unconfined compressive strength $(\mathrm{C})$ against curing time $(t)$ of the compacted soil system.

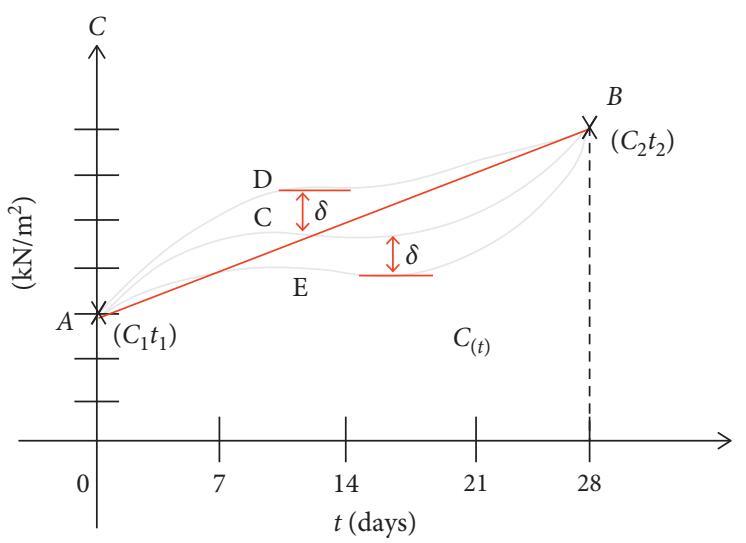

FIGURE 3: Degree of variation within the admissible variational space of typical unconfined compressive strength (C) against the curing time $(t)$ of compacted soil.

From the laws of integrals, equation (24) is substituted into equation (23) and estimated by integration by parts solution method by adopting the end conditions $t_{1=} t_{2=} 0$ as fixed endpoint problem of a constrained system, and it becomes

$$
\left[\frac{\partial F}{\partial C}-\frac{d}{\mathrm{~d} t}\left(\frac{\partial F}{\partial C^{1}}\right)\right] \varepsilon_{t} * \mathrm{~d} t=0
$$

The E-L equation of the unconfined compressive system curing time system from equation (25) presented in Figure 4 becomes

$$
\frac{\partial F}{\partial C}-\frac{d}{\mathrm{~d} t}\left(\frac{\partial F}{\partial C^{1}}\right)=0
$$

where $\mathrm{F}$ which is interchangeably used with $L$ is the Lagrange and is given by equation (16) as

$$
F=\sqrt{1+\left(C_{t}^{1}\right)^{2}}
$$

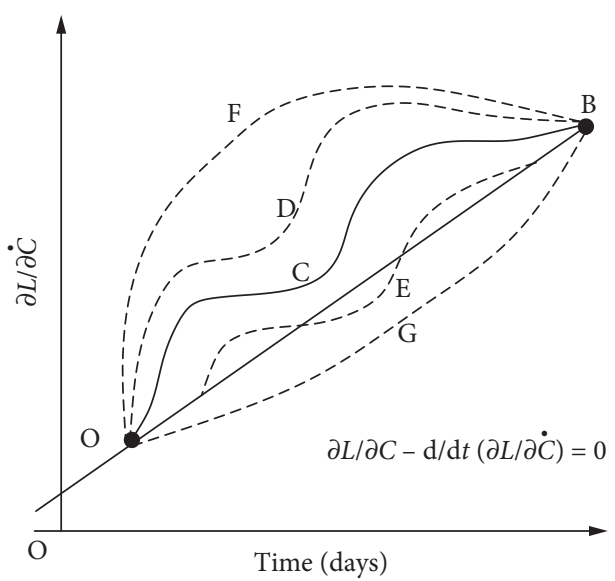

FIgURE 4: The Euler-Lagrange typical presentation of the effect of curing time on the unconfined compressive strength of compacted soil.

Equation (27) is substituted in equation (26) applying boundary conditions and solving to achieve the straight-line path of the system which lies within the AVS to get

$$
C_{t}^{1}=a t+b,
$$

where $a$ is the slope of the system path A-B and $b$ is the intercept of the system path $A-B$ on $C$ axis in $\mathrm{kN} / \mathrm{m}^{2}$. The values of a and $b$ become the boundary conditions for resolving the entire system and estimating the maximized $\mathrm{C}$ and minimized $t$.

\section{Conclusions}

$\mathrm{CoV}$ has been demonstrated as an optimization tool which can simulate the behavior of geotechnical properties of construction materials in soft or expansive soil blends as stabilized and compacted earth materials utilized in the construction industry. Various computational and artificial intelligence procedures have been in use, but the use of $\mathrm{CoV}$ in the field of geotechnical and geoenvironmental engineering is evolving. This illustrative overview work has keyed in to demonstrate that the $\mathrm{CoV}$ can be used to model systems in geotechnics for sustainable construction materials utilization and design. This has further shown that as long as the quality properties of an engineering system can be illustrated graphically, the deployment of $\mathrm{CoV}$ becomes a powerful tool to simulate the input parameters for material utilization, design, and performance monitoring purposes. The typical effect of curing time on the compressive strength of untreated or treated compacted earth material has been used to demonstrate these possibilities in geotechnical engineering. The functional changes and degrees of variation of the systems under study are harmonized using Hamilton's method for the fact that these factors are not affected by friction and gravity. Further research is suggested to study the employment of Brachistochrone solution to resolve geotechnics systems affected by friction and gravity, for example, compaction procedure where the compactive effort 
hammer drops from a measured height to effect densification of compacted soils and shear parameters evaluation.

\section{Data Availability}

The data were derived from the available literature and computational breakthrough evolved as a novel method where calculus of variations unlike before is deployed in solving geotechnical and construction material system problems.

\section{Conflicts of Interest}

The author declares that there are no conflicts of interest.

\section{References}

[1] K. C. Onyelowe, M. Iqbal, F. E. Jalal, M. E. Onyia, and I. C. Onuoha, "Application of 3-algorithm ANN programming to predict the strength performance of hydrated-lime activated rice husk ash treated soil," Multiscale and Multidisciplinary Modeling, Experiments and Design, vol. 23, no. 1, 2021.

[2] K. C. Onyelowe, M. Iqbal, and F. E. Jalal, "Smart computing models of California bearing ratio, unconfined compressive strength, and resistance value of activated ash-modified soft clay soil with adaptive neuro-fuzzy inference system and ensemble random forest regression techniques," Multiscale and Multidisciplinary Modeling, Experiments and Design, vol. $68,2021$.

[3] I. K. Andre', "Advanced calculus with applications in statistics," in Revised and Expanded, John Wiley \& Sons, Hoboken, New Jersey, 2nd edition, 2003.

[4] L. P. Lebedev, M. J. Cloud, and V. A. Eremeyev, Advanced Engineering Analysis; The Calculus of Variations and Functional Analysis with Applications in Mechanics, World Scientific Publishing Co. Pte. Ltd., Singapore, 2012.

[5] M. E. George, Calculus of Variations with Applications, Dover Publications, Inc., New York, NY, USA, 1985.

[6] L. Komzsik, Applied Calculus of Variations for Engineers, CRC PressTaylor \& Francis Group, Boca Raton, FL, USA, 2009.

[7] F. Clarke, Functional Analysis, Calculus of Variations and Optimal Control, Springer, New York, NY, USA, 2013.

[8] J. A. Burns, Introduction to The Calculus of Variations and Control with Modern Applications, CRC Press Taylor \& Francis Group, Boca Raton, FL, USA, 2014.

[9] I. Papst, "A biological application of the calculus of variations," The Waterloo Mathematics Review, vol. 6, pp. 3-16, 2012.

[10] J. M. BALL, "The calculus of variations and materials science," Quarterly of Applied Mathematics, vol. 106, no. 4, pp. 719-740, 1998.

[11] U. L. Rohde, G. C. Jain, and A. K. Ghosh, Introduction to Differential Calculus; Systematic Studies with Engineering Applications for Beginners, John Wiley \& Sons, Hoboken, NJ, USA, 2012.

[12] U. L. Rohde, G. C. Jain, and A. K. Ghosh, Introduction to Integral Calculus; Systematic Studies with Engineering Applications for Beginners, John Wiley \& Sons, Hoboken, NJ, USA, 2012.

[13] K. C. Onyelowe, "Bearing capacity of footing on slope by variational calculus," Research Journal of Engineering and Applied Sciences, (RJEAS), USA, vol. 1, no. 1, 2012.
[14] K. C. Onyelowe, "Mathematical advances in soil bearing capacity," Electronic Journal of Geotechnical Engineering, vol. 22, no. 12, pp. 4735-4743, 2017, http://www.ejge.com.

[15] K. Onyelowe, G. Alaneme, C. Igboayaka et al., "Scheffe optimization of swelling, California bearing ratio, compressive strength, and durability potentials of quarry dust stabilized soft clay soil," Materials Science for Energy Technologies, vol. 2, no. 1, pp. 67-77, 2019.

[16] K. C. Onyelowe and V. D. Bui, "Predicting strength behavior of stabilized lateritic soil- ash matrix using regression model for hydraulically bound materials purposes," International Journal of Pavement Research and Technology, vol. 40, 2018.

[17] J. F. Tsai, J. G. Carlson, D. Ge, Y. C. Hu, and J. Shi, "Optimization theory, methods, and applications in engineering," Mathematical Problems in Engineering, vol. 2014, Article ID 319418, 2014 